\title{
Sphincter-Preserving Surgery for Rectal Cancer: A Controversial Measure of Quality
}

\author{
Samantha Hendren, MD, MPH and Zaid Abdelsattar, MD \\ Department of Surgery, University of Michigan, Ann Arbor
}

In the last 25 years, the treatment for rectal cancer has improved markedly. Multimodality therapy and higherquality surgery have resulted in low rates of local recurrence (previously a terrible problem) as well as impressive rates of long-term survival that sometimes exceed those for colon cancer. ${ }^{1}$ Credit must be given to the important treatment trials and to the quality assessment and improvement programs in Europe and elsewhere that have raised the bar of care quality for rectal cancer. ${ }^{2}$ Thus it is appropriate that we turn our attention to preserving function and quality of life for these patients.

Rectal cancer is indeed a condition for which treatment may result in extreme long-term effects on patient's lives. Bowel function, sexual and urinary function, body image, and work and social productivity all are potentially affected for patients rendered cancer free. ${ }^{3,4}$ An oncologic success may be a quality-of-life failure.

In this Annals of Surgical Oncology issue, Mohammed and colleagues focus on the most apparent functional aspect of rectal cancer treatment: sphincter-preserving surgery (SPS). ${ }^{5}$ In their study, they compare rates of SPS between veteran patients identified in the Veterans Affairs (VA) Central Cancer Registry and patients in the national Surveillance, Epidemiology, and End Results (SEER) registry during the period 1995-2010. They find that the rates of SPS have increased in both populations and that VA rates are now a bit higher than national rates, at

This is an invited editorial to the article available at doi: $10.1245 /$ s10434-014-4101-z

(C) Society of Surgical Oncology 2014

First Received: 5 September 2014;

Published Online: 17 September 2014

S. Hendren, MD, MPH

e-mail: hendren@med.umich.edu
$79.3 \%$. This is seen as a triumph for VA care quality improvement given that older research had indicated higher abdominoperineal resection (APR) rates for the VA than for the private sector. The study then focuses on patients with early-stage rectal cancer (nonirradiated, pathologic stages 0 and 1) and finds that $18 \%$ of these patients undergo an APR. In their discussion, the authors plead for more SPS in this group.

The strength of this study is its use of data from parallel national tumor registries, which allows for accurate staging and direct comparisons. It is also a strength that these data are recent because SPS rates are changing with time. However, in interpreting these results, it is important to acknowledge the weaknesses inherent in the data. First, tumor registries lack key clinical information including tumor location and patient's sphincter function. This weakness is common to most studies on this topic. Second, some patients may be miscategorized as undergoing SPS when their surgery in fact resulted in a permanent colostomy because Hartmann's-type procedures are included in the procedure category for anterior resection. Third, radiation therapy information in tumor registries is known to be inaccurate, often miscategorizing patients who have had radiation therapy as having not received it. ${ }^{6}$ These data limitations may have affected the accuracy of SPS rates and the analysis of early-stage rectal cancers. If prior radiation therapy or surgery had pathologically downstaged these cases, they may not have been overtreated at all.

Notwithstanding these limitations, this is an important study of cancer care in the national VA health care system. The authors are almost certainly correct in reporting that quality of cancer care has improved in the VA, which has moved toward increased subspecialty care in recent years and has a strong health care quality research group.

A minor point in the study is worth mentioning: the adequacy of lymph node examination remains an opportunity for improvement in the VA, with an average of 9.5 
lymph nodes examined, compared with 11.5 for rectal cancer patients in the SEER database in $2005 .^{7}$ These results are concordant with prior research showing lower numbers of lymph nodes examined in VA hospitals for colon cancer. ${ }^{8}$ Regardless, we believe this study highlights meaningful improvement in the use of modern surgical techniques in the VA.

The author's emphasize their finding of an $18 \%$ APR rate for apparently early-stage rectal cancers that "on paper" might have been appropriately treated with local excision. This finding deserves further study. We suggest that it is unknown whether these patients were in fact overtreated. Alternative explanations for the use of APR in these patients include (1) neoadjuvant therapy inaccurately omitted from the tumor registry, as mentioned earlier, and (2) prior nonradical surgery (via a transanal approach) that resulted in positive margins, recurrence, or unfavorable characteristics necessitating further surgery.

We hope the authors perform a follow-up study with a detailed chart review to help clinicians understand the use of APR in this population. Such a study would be a valuable contribution to the literature on this topic, which in general is limited by the lack of nuanced clinical data. It is difficult to propose quality improvement strategies without a deeper understanding of why APR is performed in such cases.

These uncertainties of the data raise the question: should SPS be used as a quality measure-for surgeons, hospitals, or institutions nationally? The surgical community has taken for granted that this occurs, but no data source exists that can accurately differentiate those patients eligible for SPS from those who for anatomic, functional, or oncologic reasons are not. As the authors point out, referral patterns also make between-provider comparisons difficult. Our own research, with a more in-depth source of data including tumor location and abstraction of some reasons for non-SPS from charts, still fails to understand why SPS is not performed and how therefore to improve. ${ }^{9}$ We propose that research into surgeon's practices and motivations and the clinical details of cases are essential before SPS can be used as a quality measure.

Finally, this discussion to date has not included the most fundamental problem with promoting more SPS for rectal cancer-the patient-preference-sensitive trade-off between oncologic success and quality of life. We must be vigilant to avoid repeating the mistakes of the past (i.e., performing inadequate surgery that leads to higher rates of unsalvageable local recurrences). ${ }^{10}$ We await more evidence, such as the long-term results of the ACOSOG Z6041 trial, before we can safely combine local excision with chemoradiation in more cases, thus achieving the dual objective of oncologic and SPS success. However, until there is increased clarity about how to select invasive cancer patients for nonradical surgery (beyond the small, welldifferentiated $\mathrm{T} 1$ rectal cancers for which it is the standard of care), caution must be exercised in promoting fewer mesorectal excisions, which have resulted in the oncologic successes we currently enjoy.

\section{REFERENCES}

1. Nedrebo BS, Soreide K, Eriksen MT, et al. Survival effect of implementing national treatment strategies for curatively resected colonic and rectal cancer. Br J Surg. 2011;98:716-23.

2. van Gijn W, van de Velde CJ. 2010 SSO John Wayne clinical research lecture: rectal cancer outcome improvements in Europe: population-based outcome registrations will conquer the world. Ann Surg Oncol. 2010;18:691-6.

3. van den Brink M, van den Hout WB, Kievit J, Marijnen CA, et al. The impact of diagnosis and treatment of rectal cancer on paid and unpaid labor. Dis Colon Rectum. 2005;48:1875-82.

4. Wiltink LM, Chen TY, Nout RA, et al. Health-related quality of life 14 years after preoperative short-term radiotherapy and total mesorectal excision for rectal cancer: report of a multicenter randomised trial. Eur J Cancer. 2014;50(14):2390-8.

5. Mohammed S, Anaya DA, Awad SS, et al. Sphincter-preservation rates following radical resection for rectal cancer in the United States veteran population: opportunity for improvement in early disease. Ann Surg Oncol. 2014. doi:10.1245/s10434-0144101-z.

6. Walker GV, Giordano SH, Williams M, et al. Muddy water? Variation in reporting receipt of breast cancer radiation therapy by population-based tumor registries. Int J Radiat Oncol Biol Phys. 2013;86:686-93.

7. Chou JF, Row D, Gonen M, et al. Clinical and pathologic factors that predict lymph node yield from surgical specimens in colorectal cancer: a population-based study. Cancer. 2010;116:256070.

8. Bilimoria KY, Bentrem DJ, Stewart AK, et al. Lymph node evaluation as a colon cancer quality measure: a national hospital report card. J Natl Cancer Inst. 2008;100:1310-7.

9. Abdelsattar ZM, Wong SL, Birkmeyer NJ, et al. Multi-institutional assessment of sphincter preservation for rectal cancer. Ann Surg Oncol. 2014. doi:10.1245/s10434-014-3882-4.

10. Madoff RD. Total mesorectal neglect in the age of total mesorectal excision. J Clin Oncol. 2013;31:4273-5. 